\title{
Steroids for Community-Acquired Pneumonia? Not in My House!
}

\section{To the Editor:}

In response to the paper by PR Bauer and VN lyer (1) entitled "Corticosteroids and Influenza A Associated Acute Respiratory Distress Syndrome" published in the Southwest Journal of Pulmonary and Critical Care (SWJPCC) on November 18, 2016. That paper presents a single case report of a patient with community-acquired pneumonia in Arizona attributed to influenza A. The patient was treated with steroids and improved. For a variety of reasons, a case report like this should not be published unless accompanied by a note pointing out the implications of such a report. The audience of SWJPCC is generally going to be made up of two groups, mainly from Arizona. Those with experience who would say that the treating physicians were lucky (but in the back of their minds this case might give them a reason to use steroids in similar circumstances) and those with little or no experience, who will say the next time I see a patient with a non-bacterial pneumonia who is not doing well, I am going to treat that patient with steroids. The latter interpretation is the reason for this Letter to the Editor to point out the problems.

Why am I in a position to make judgments in this clinical situation? Because I was in private practice in Phoenix ("my house") as a Board Certified Infectious Diseases specialist for over 25 years. I estimate that I followed about 10,000 patients hospitalized with various types of pneumonia. I have not published any papers on pneumonia in Arizona, but I have a lot of experience in treating pneumonias in Phoenix, the sixth largest city in the United States. In addition, I was the Infectious Disease Society of America (IDSA) "Clinician of the Year in 2007" which suggests that I have some experience and knowledge with the infectious disease issues at hand. There is a general clinical observation made by me over the years about the use of steroids in pneumonias of unknown cause - that is the steroid-treated patients initially seem to improve for a few days, irrespective of the cause. Unfortunately those starting the steroids only remember how the patient seemed to improve with steroids, but forget that the patient dies about a week later. The treating physicians only remember the initial improvement associated with the steroids and not the fact the patient ultimately died.

A few years ago I reviewed the medical records of six patients with meningitis due to Coccidioides. Of the six, two had been seen previously as outpatients for a pneumonia and given steroids (for unclear reasons), in addition to empirical antibiotics. I knew the hospitalists who cared for those two patients. They were good physicians, but new to Phoenix and had received their training outside of Arizona. These observations would be difficult to make unless you had experience in this part of the world where there are lots of people and Coccidioides together in one place, more than anywhere else in the world. I experienced similar issues myself of "occult" Coccidioides when I began practice in Phoenix in 1980. It has been my idea that all physicians who get a license to practice in Arizona should show proficiency in coccidioidomycosis.

The patient described in this Journal's case report was felt to have influenza. Please note however, that he had tested negative for influenza twice before a culture was 
positive for influenza $A$. This supports the possibility that the influenza detection may have been incidental to some other underlying etiology. In my opinion, to publish a single case report of influenza pneumonia treated with steroids there should be lung biopsy evidence consistent with influenza pneumonia. Publishing a single case report, only perpetuates the idea that using steroids might be beneficial in influenza pneumonia. In controlled studies on the use of steroids there has not been clear evidence that they are therapeutic in influenza pneumonia. The authors prefer to call it a "controversy" to justify their use of steroids, but if this patent had died of coccidioidomycosis, what would be the controversy then? How much to pay the plaintiff! Please note the author's affiliation is listed as Rochester, Minnesota an area not known to have endemic Coccidioides.

There are three antiviral agents that can be used for serious influenza virus infections: 1) Oral oseltamivir (Tamiflu) which was used in this patient. In critically ill patients a nasogastric tube may be needed to administer the oral agent. Starting the drug within 72 hours of onset is optimal, but the drug can still have some effectiveness if started 4-5 days later; 2) Intravenous peramivir (Rapivab) was recently approved (12/2014) for use against influenza. I prefer the intravenous formulation in critically ill patients; 3 ) There is an intravenous version of inhaled zanamivir (Relenza) which can be obtained on an investigational basis. The sooner any of these agents are started the better the outcome. In any patient critically ill with pneumonia during flu season, any of these agents should be started empirically even before the influenza studies are completed.

It should be noted that the patient in the case report received both oseltamivir and methylprednisolone. One could make the case that since steroids have not been shown in clinical studies to be effective in influenza pneumonia, that the clinical response may have been to the oseltamivir. Often patients with influenza pneumonia will have rapid progression to respiratory failure before the antivirals are considered. Starting oseltamivir or peramivir empirically on only the suspicion of a viral pneumonia is more likely to be beneficial than the empirical use of antibacterial antibiotics.

Young physicians recently out of training usually have little experience with the use of antivirals in critically ill patients. In my opinion, the only benefit of publishing a single case of influenza A pneumonia is to have a companion rebuttal and discussion of the issues. Hopefully these observations will give pause to the Arizona clinician's next patient who appears to have a non-bacterial pneumonia - think of the early use of antivirals and the diagnosis of Coccidioides, not the use of steroids.

Tim Kuberski, MD, FIDSA

Maricopa Medical Center

Department of Medicine, Infectious Diseases

Phoenix, Arizona 


\section{Reference}

1. Bauer PR, lyer VN. Corticosteroids and influenza $A$ associated acute respiratory distress syndrome. Southwest J Pulm Crit Care. 2016;13(5):248-51. [CrossRef]

\section{The Authors Reply:}

We read with interest the letter to the Editor by Dr. Kuberski and acknowledge his concern. We intended to publish this case report for the exact same reason that triggered his worries. How can someone use corticosteroids in Influenza when their benefits are controversial $(1,2)$ ? Here are some elements of responses. First, Influenza affects Arizona like every other State so it is relevant to the readers in this part of the Country as well. Second, in spite of their controversial benefits, corticosteroids are commonly prescribed in Influenza-related illnesses (3). Third, due to its low sensitivity, albeit high specificity, diagnostic testing for influenza maybe negative in the upper airways (e.g. nasal swab) and positive in the lower airways (e.g. broncho-alveolar lavage) and therefore antiviral treatment can be delayed and outcome jeopardized like in our case. Fourth, the benefit of an anti-influenza agent is optimal if initiated within 48 hours after symptoms onset. Fifth, biopsy and electronic microscopy are not recommended to confirm the diagnosis of Influenza and the plan of care in this case assumed a high likelihood of severe Influenza with delayed diagnosis and therefore delayed treatment.

In spite of the severity of the presentation to our Institution, this patient's condition improved dramatically, rapidly and definitively. We certainly cannot conclude that corticosteroids made the difference but the fact of the matter is that this patient did not need prolonged ventilation or extracorporeal membrane oxygenation. The use of corticosteroids in acute respiratory distress syndrome (ARDS) has been suggested long time ago but indeed was not associated with better long term outcome (4). That particular study was conducted between April 1997 and April 2002 that is before the era of lung volume protection strategy (published in 2000) and may have been confounded by the occurrence of ventilator associated lung injury and nosocomial infection (5). With the new Berlin definition of ARDS (6), not every ARDS is associated with diffuse alveolar damage for which corticosteroids have no known benefit; organizing pneumonia is also frequently encountered in ARDS which may be sensitive to corticosteroids (7). Lastly, the recent study on corticosteroids in community acquired pneumonia, which by the way included 27 cases of Influenza, demonstrated overall a shorter time to clinical stability without an increase in complications, like in our case (8). At the end, this patient with moderate ARDS secondary to Influenza made a prompt and uncomplicated recovery, despite delayed treatment with antiviral agent and the adjunction of corticosteroids. We were surprised and thrilled by the patient's fast and full recovery and wanted to share our experience and revisit the role of corticosteroids in this particular context.

Philippe R Bauer, MD, PhD, and Vivek N. lyer, MD Pulmonary and Critical Care Medicine Mayo Clinic 
Rochester, MN USA

\section{References}

1. Rodrigo C, Leonardi-Bee J, Nguyen-Van-Tam J, Lim WS. Corticosteroids as adjunctive therapy in the treatment of influenza. Cochrane Database Syst Rev. 2016 Mar 7;3:CD010406. [CrossRef] [PubMed]

2. Shafiq M, Mansoor MS, Khan AA, Sohail MR, Murad MH. Adjuvant steroid therapy in community-acquired pneumonia: a systematic review and meta-analysis. J Hosp Med. 2013 Feb;8(2):68-75. [CrossRef] [PubMed]

3. Delaney JW, Pinto R, Long J, et al. The influence of corticosteroid treatment on the outcome of influenza A(H1N1pdm09)-related critical illness. Crit Care. 2016 Mar 30;20:75. [CrossRef] [PubMed]

4. Meduri GU, Golden E, Freire AX, et al. Methylprednisolone infusion in early severe ARDS: results of a randomized controlled trial. Chest. 2007 Apr;131(4):954-63. [CrossRef] [PubMed]

5. The Acute Respiratory Distress Syndrome Network. Ventilation with lower tidal volumes as compared with traditional tidal volumes for acute lung injury and the acute respiratory distress syndrome. N Engl J Med. 2000 May 4;342(18):1301-8. [CrossRef] [PubMed]

6. Ranieri VM, Rubenfeld GD, Thompson BT, et al. Acute respiratory distress syndrome: the Berlin Definition. JAMA. 2012 Jun 20;307(23):2526-33. [CrossRef] [PubMed]

7. Thille AW, Esteban A, Fernández-Segoviano P, et al. Comparison of the Berlin definition for acute respiratory distress syndrome with autopsy. Am J Respir Crit Care Med. 2013 Apr 1;187(7):761-7. [CrossRef] [PubMed]

8. Blum CA, Nigro N, Briel M, et al. Adjunct prednisone therapy for patients with community-acquired pneumonia: a multicentre, double-blind, randomised, placebocontrolled trial. Lancet. 2015;385(9977):1511-8. [CrossRef] [PubMed] 